\begin{tabular}{|l|l|}
\hline $\mathrm{AD}-1$ & 1 \\
\hline
\end{tabular}

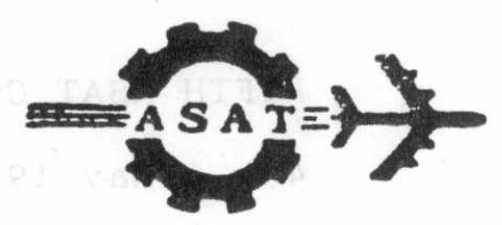

\title{
NUMERICAL SOLUTION OF THE UNSTEADY INCOMPRESSIBLE NAVIER- STOKES EQUATIONS IN GENERALIZED NON-ORTHOGONAL CURVILINEAR CO-ORDINATES
}

\author{
M.M. ABDELRAHMAN* and A.M. AL-BAHI*
}

\begin{abstract}
A method based on the vorticity stream function formulation is used for the solution of the unsteady incompressible two-dimensional laminar viscous flow problems. Vorticity transport and stream function equations are written in generalized curvilinear non-orthogonal coordinate system, and discretized using an implicit finite difference approximation. A second order accurate scheme with a stabilizing correction splitting method as an ADI technique is used. Both conservative and non-conservative forms of the vorticity transport equation are considered and the problems associated with different treatments of boundary conditions are addressed. Several test cases, including rectangular cavity flow problem, flow around circular cylinder and flow past an isolated airfoil at moderate angles of attack, are illustrated.
\end{abstract}

\section{INTRODUCTION}

Solutions of Navier-Stokes equations provide an excellent description of viscous Newtonian fluid motions. Due to the coupling problem and the non linearity, only numerical approximations to these solutions can be

\footnotetext{
*Aeronautical Eng. Dept., King Abdulaziz University, P.O.Box 9027, Jeddah 21413, Saudi Arabia.
} 
obtained. Such numerical solutions, which require the full exploitation of the latest computer technology and integration procedures, can generally be grouped into three categories:

- Methods based on artificial compressibility,

- Methods using pressure iteration, and

- Methods based on vorticity formulation.

In the artificial compressibility methods [1], the elliptic-parabolic nature of the unsteady incompressible Navier Stokes equations is changed to a hyperbolicparabolic one, by adding an artificial time derivative of the pressure to the continuity equation. This allows utilising the highly developed algorithms of compressible flow to incompressible problems. Although these methods are normally limited to steady state flow problems; an extension to unsteady flows by dual time stepping has been successfully used by several authors [2-4]. In this dual time method, artificial compressibility technique is applied at each time step. Consequently, the drawback of this method is the large computational effort to establish a converged solution each time step .

In the methods based on pressure iteration, the velocities are calculated from the momentum equations with an estimated pressure gradient, then the pressure is imposed by an iterative procedure for each time step. Another way to derive a pressure equation is to form a Poisson equation by taking the divergence of the momentum equations. The Marker and Cell method, (MAC), proposed by Harlow and Welsh [5] and the Projection method presented by Chorin [1] are prototypes of such a method. The main drawbacks associated with the solution of the Navier Stokes equations using pressure-velocity formulation are the lack of the boundary conditions for the pressure and the necessity of satisfying the zero velocity divergence at any time.

In the vorticity formulation methods, the pressure is replaced by the vorticity vector, and the vorticity transport equation is solved instead of the momentum equations. The continuity equation, on the other hand, can be satisfied in two different ways. The first is the vorticity-velocity approach proposed by Fasel [6] and used by several authors [7-8], in which care has to be taken for vorticity boundary conditions in order to satisfy the conservation of mass. The second way is to use a stream function formulation. This approach, which is 
for two-dimensional cases, can be extended to three dimensions by defining a vector potential instead of the stream function. This vector potential has to be calculated from the three Poisson equations. An example of such threedimensional formulation is described in [9].

In this paper the Navier Stokes equations are studied in the vorticity stream function formulation which appears to be the optimal choice for twodimensional problems, mainly from storage point of view.

\section{GOVERNING EQUATIONS}

Navier Stokes equations for incompressible viscous flows can be written in the vector form as [10 ]

$\nabla^{2} \Psi=-\omega$

$\frac{\partial \omega}{\partial t}+(\mathrm{V} \cdot \nabla) \omega=\frac{1}{R_{e}} \nabla^{2} \omega$

$\mathbf{V}=\nabla \wedge \Psi$

where $\boldsymbol{\omega}$ is the vorticity vector, $\Psi$ is the stream function vector, $\mathbf{V}$ is the velocity vector and $\mathrm{Re}$ is the Reynolds number. The system of equations (1) is made dimensionless using a reference velocity and length.

The system of equations (1) becomes most interesting for the two-dimensional case where the stream function vector and the vorticity vector have only one component normal to the plane of flow. The equations (1.a) and (1.b) become scalar equations and instead of solving a system of six equations in the general three-dimension case, a two- equation system is to be solved.

Eqn. (1.b), which represents the momentum equation, is in a non conservative form, and can be written in the following conservative form: 
$\frac{\partial \omega}{\partial \mathrm{t}}+\nabla \cdot(\mathrm{V} \omega)=\frac{1}{\operatorname{Re}} \nabla^{2} \omega$

where the convective term in Eqns (1.b) and (2) are equivalent for a zero divergence velocity field i.e. incompressible flow.

An important feature of the stream-function vorticity formulation is that the pressure is no longer explicit in the equation. If however, the pressure is needed in the flow field, the following poisson equation must be solved

$\nabla^{2} p=2\left(\frac{\partial u}{\partial x} \frac{\partial v}{\partial y}-\frac{\partial v}{\partial x} \frac{\partial u}{\partial y}\right)$

where $\mathbf{u}$ and $\mathbf{v}$ are the velocity components in the Cartesian coordinate system $(\mathbf{x}, \mathbf{y})$.

Now let us introduce the generalized non-orthogonal curvilinear co-ordinates $\eta_{1}$ and $\eta_{2}$, given by:

$\eta_{1}=\eta_{1}(\mathrm{x}, \mathrm{y}, \mathrm{t}) \quad, \quad \eta_{2}=\eta_{2}(\mathrm{x}, \mathrm{y}, \mathrm{t})$

with the covariant base vectors $\alpha_{1}$ and $\alpha_{2}$, tangent to $\eta_{1}$ and $\eta_{2}$, respectively, and defined by:

$\alpha_{1}=x_{\eta_{1}} \overline{\mathbf{i}}+\mathbf{y}_{\eta_{1}} \overline{\mathbf{j}} \quad, \quad \alpha_{2}=x_{\eta_{2}} \overline{\mathbf{i}}+\mathbf{y}_{\eta_{2}} \overline{\mathbf{j}}$

The governing equations, (1.a) and (2), are transformed in this generalized coordinate system to:

$-\sqrt{\mathrm{g}} \omega=\frac{\partial}{\partial \eta_{\mathrm{j}}}\left(\mathrm{g}^{\mathrm{ij}} \sqrt{\mathrm{g}} \frac{\partial \Psi}{\partial \eta_{\mathrm{i}}}\right)$

$\sqrt{\mathrm{g}} \frac{\partial \omega}{\partial t}+\frac{\partial}{\partial \eta_{\mathrm{i}}}\left(\sqrt{\mathrm{g}} \mathrm{V}^{\mathrm{i}} \omega\right)=\frac{1}{\operatorname{Re}} \frac{\partial}{\partial \eta_{\mathrm{j}}}\left(\mathrm{g}^{\mathrm{ij}} \sqrt{\mathrm{g}} \frac{\partial \omega}{\partial \eta_{\mathrm{i}}}\right)$

where Einstein tensor notation is used with summation over repeated indices. 
In Eqns. (6) and (7), the transformation metric, $\mathrm{g}$, is given by.

$$
g=\left|\begin{array}{ll}
g_{11} & g_{12} \\
g_{12} & g_{22}
\end{array}\right|=\left(x_{\eta_{1}} y_{\eta_{2}}-x_{\eta_{2}} \mathbf{y}_{\eta_{1}}\right)^{2}
$$

with the covariant metric components, $\mathbf{g}_{\mathbf{i j}}$ (for $\mathrm{i}=1,2$ and $\mathrm{j}=1,2$ ):

$$
\mathbf{g}_{i j}=\alpha_{i} \cdot \alpha_{j}=x_{\eta_{1}} x_{\eta_{1}}+\mathbf{y}_{\eta_{1}} \mathbf{y}_{\eta_{1}}
$$

The contravariant metric components gij are calculated by.

$$
\left[g^{i j}\right]=\left[g_{i j}\right]^{-1} \Rightarrow g^{11}=\frac{g_{22}}{g}, g^{12}=-\frac{g_{12}}{g}, g^{22}=\frac{g_{11}}{g}
$$

And the contravariant velocity components are expressed by

$$
\mathrm{V}^{1}=\frac{\partial \Psi}{\partial \eta_{2 .}} \frac{1}{\sqrt{\mathrm{g}}} \quad, \quad \mathrm{V}^{2}=-\frac{\partial \Psi}{\partial \eta_{1}} \frac{1}{\sqrt{\mathrm{g}}}
$$

The transformed partial differential equations are of the same form and type as the original equations, and are more complicated only in the sense of having variable coefficients, cross derivatives, and more terms.

\section{NUMERICAL ALGORITHM}

\section{Finite Difference Discretization}

The computation of the stream function and the vorticity are carried out using a second order finite difference approximation. The discretized continuity and momentum equations can be written as:

$\sqrt{g_{i, j}} \cdot \omega_{i, j}+T_{1}+T_{2}+T_{3}+T_{4}=0$ 


$$
\begin{aligned}
& \sqrt{g_{i, j}} \frac{\omega^{n+1}{ }_{i, j}-\omega^{n}{ }_{i, j}}{\Delta t} \\
& +\frac{V^{1}}{2}\left\{\left(1-\varepsilon_{1}\right) \frac{\omega^{n+1}{ }_{i+1, j}-\omega^{n+1}{ }_{i, j}}{\Delta \eta_{1}}+\left(1+\varepsilon_{1}\right) \frac{\omega^{n+1}{ }_{i, j}-\omega^{n+1}{ }_{i-1, j}}{\Delta \eta_{1}}\right\} \\
& +\frac{V^{2}}{2}\left\{\left(1-\varepsilon_{2}\right) \frac{\omega^{n+H_{i, j+1}}-\omega^{n+1}{ }_{i, j}}{\Delta \eta_{2}}+\left(1+\varepsilon_{2}\right) \frac{\omega^{n+1}{ }_{i, j}-\omega^{n+1}{ }_{i, j-1}}{\Delta \eta_{2}}\right\} \\
& =\frac{1}{R e}\left[T^{1}+T^{2}+T^{3}+T^{4}\right]
\end{aligned}
$$

where,

$\mathbf{T}_{\mathbf{1}}, \mathbf{T}_{\mathbf{2}}, \mathbf{T}_{\mathbf{3}}$ and $\mathbf{T}_{\mathbf{4}}$ (in Eqn. 12) and $\mathbf{T}^{\mathbf{1}}, \mathbf{T}^{\mathbf{2}}, \mathbf{T}^{\mathbf{3}}$ and $\mathbf{T}^{\mathbf{4}}$ (in Eqn. 13) represent the discretized form, of the right hand side terms in Eqns. (6) and (7) respectively, using a central space difference. For example $\mathbf{T}_{\mathbf{1}}$ and $\mathbf{T}^{\mathbf{1}}$ are expressed by:

$$
\begin{aligned}
T_{1} & =\frac{\left(g^{11} \sqrt{g}\right)_{i-1 / 2, j}}{\left(\Delta \eta_{1}\right)^{2}} \Psi_{i-1, j} \frac{\left[\left(g^{11} \sqrt{g}\right)_{i+1 / 2, j}+\left(g^{11} \sqrt{g}\right)_{i-1 / 2, j}\right]}{\left(\Delta \eta_{1}\right)^{2}} \Psi_{i, j} \\
& +\frac{\left(g^{11} \sqrt{g}\right)_{i+1 / 2, j}}{\left(\Delta \eta_{1}\right)^{2}} \Psi_{i+1, j}
\end{aligned}
$$

$$
\begin{aligned}
T^{1} & =\frac{\left(g^{11} \sqrt{g}\right)_{i-1 / 2, j}}{\left(\Delta \eta_{1}\right)^{2}} \omega_{i-1, j} \frac{\left[\left(g^{11} \sqrt{g}\right)_{i+1 / 2, j}+\left(g^{11} \sqrt{g}\right)_{i-1 / 2, j}\right]}{\left(\Delta \eta_{1}\right)^{2}} \omega_{i, j} \\
& +\frac{\left(g^{11} \sqrt{g}\right)_{i+1 / 2, j}}{\left(\Delta \eta_{1}\right)^{2}} \omega_{i+1, j}
\end{aligned}
$$

In these equations $\mathrm{n}$ is the iteration index and $\varepsilon_{1}$ and $\varepsilon_{2}$ are, respectively, the signs of the contravariant velocity components $\mathbf{V}^{\mathbf{1}}$ and $\mathbf{V}^{\mathbf{2}}$ which are approximated by: 


$$
V^{1}=\frac{1}{\sqrt{g}}\left[\frac{\Psi_{i, j+1}-\Psi_{i, j-1}}{2 \Delta \eta_{2}}\right] \quad, \quad V^{2}=\frac{-1}{\sqrt{g}}\left[\frac{\Psi_{i+1, j}-\Psi_{i-1, j}}{2 \Delta \eta_{1}}\right]
$$

The metric derivatives are calculated by:

$$
\begin{aligned}
& \mathbf{x}_{\eta_{1}}=\frac{\partial \mathbf{x}}{\partial \eta_{1}}=\frac{\mathbf{x}_{i+1 / 2, j}-\mathbf{x}_{\mathrm{i}-1 / 2, j}}{\Delta \eta_{1}}, \mathbf{y}_{\eta_{1}}=\frac{\partial \mathbf{y}}{\partial \eta_{1}}=\frac{\mathbf{y}_{i+1 / 2, j}-y_{i-1 / 2, j}}{\Delta \eta_{1}} \\
& \mathbf{x}_{\eta_{2}}=\frac{\partial \mathbf{x}}{\partial \eta_{2}}=\frac{\mathbf{x}_{i, j+1 / 2}-x_{i, j-1 / 2}}{\Delta \eta_{2}}, y_{\eta_{2}}=\frac{\partial y}{\partial \eta_{2}}=\frac{y_{i, j+1 / 2}-y_{i, j-1 / 2}}{\Delta \eta_{2}}
\end{aligned}
$$

These metric derivatives should be evaluated directly from the difference between grid points. It is, therefore, necessary to have coordinate values available at inter-grid points. The Jacobian of the transformation and covariant and contravariant metric components are calculated using Eqns. (8), (9) and (10).

A very efficient implicit technique for solving the system, in multidimensional, is the stabilising correction splitting method. This method, which improves convergence, is now extensively used in both its original form proposed by Peaceman and Rachford [11] and in its generalized form [12]. Without imposing boundary conditions this splitting $A D I$ scheme is unconditionally stable in the linearized case.

\section{Boundary Conditions}

The problem associated with the above system of governing equations is an initial boundary value problem. In terms of the stream function, the boundaries has to satisfy the total flux condition. Meanwhile the boundary condition for the vorticity must be evaluated using Eqn. 12. This coupling between the vorticity and the second derivatives of the stream function at the boundary represents the main difficulty in this vorticity-stream function approach. In this case, it is necessary to evaluate the second derivatives of $\psi$ with a degree of accuracy sufficient to obtain an acceptable solution of the vorticity. 
Using a body fitted coordinate system with $\eta_{2}=$ constant on the surface of the body, $T_{1}$ will be zero. On the other hand $T_{2}, T_{3}$ and $T_{4}$ can be calculated by a second order central difference approximation similar to the internal grid points, where the values of $\boldsymbol{\Psi}_{i, j-1}, \boldsymbol{\Psi}_{i-1, j-1}$ and $\boldsymbol{\Psi}_{i+1, j-1}$ are the values outside the computational domain (image points).

In the present work two different methods are used to calculate the values of the stream function at the image points.

- In the first method, the image points are calculated using a least square fourth order polynomial fitting with sufficient number of points near the boundary

- In the second method, image points are calculated using the Taylor series expansion with second order approximations and only three points such that:

$\Psi_{k, j-1}=\frac{-\Psi_{k, j+2}+6 \cdot \Psi_{k, j+1}-3 \cdot \Psi_{k, j}}{2}$ for $k=i-1, i$ and $i+1$

At the inflow boundary both the stream function and the vorticity distributions are prescribed. At the outflow boundary the value of the stream function and the vorticity are determined such that the velocity component normal to the free stream and the down stream vorticity are conserved in the flow direction.

\section{Pressure and Shear Stress}

If only the pressure on the solid boundary is desired, it is not necessary to solve Eqn. 3 over the entire field. Instead, a simpler equation can be obtained by applying the tangential momentum equation to the fluid adjacent to the wall surface. This equation can be written in the vector form as:

$\nabla \mathbf{p}=-\frac{1}{\mathbf{R}_{e}}[\nabla \wedge \omega]$

In the general curvilinear non-orthogonal co-ordinates system this equation can be written after discretization as: 
$\frac{p_{i+1, j}-p_{i, j}}{\Delta \eta_{1}}=-\frac{1}{R_{e}} \sqrt{g} \cdot\left\{g^{12} \frac{\left[\omega_{i+1, j}-\omega_{i, j}\right]}{\Delta \eta_{1}}+g^{22} \frac{\left[-3 \omega_{i, j}+4 \omega_{i, j+1}-\omega_{i, j+2}\right]}{\Delta \eta_{2}}\right\}$

where $\eta_{1}$ is the coordinate line adjacent to the solid boundary. In order to apply the previous equation to find the pressure at all points on the wall surface, the pressure must be known for at least one point on the solid boundary. Calculations were started from the stagnation point whose pressure is known.

The shear stress at the solid boundary can be written in the generalized nonorthogonal curvilinear co-ordinates as

$$
\tau=\frac{1}{R_{e} \sqrt{g^{22}}} \frac{\partial}{\partial \eta_{2}}\left[\frac{1}{\sqrt{g^{22}}}\left(g^{22} \frac{\partial \Psi}{\partial \eta_{2}}+g^{12} \frac{\partial \Psi}{\partial \eta_{1}}\right)\right]
$$

\section{TEST CASES}

To illustrate the present numerical code, three test cases are considered.

The first test case concerns the cavity flow problem. This popular example for comparing numerical methods is shown in Fig. (1). The fluid velocity is zero on the three sides of the square $(B C, C D, D A)$. A velocity profile on the side $A B$ proposed by Bourcier and Francois [10] is considered in the following form:

$u(x)=-16 x^{2}(1-x)^{2}$

Computations was performed for the case of $(\mathbf{R e}=200)$. A rectangular computational grid of $21 \times 21$ points was generated using a stretching function to cluster more points near the side $A B$. Figs. (2) and (3) show the steady state streamlines and the isovorticity lines.

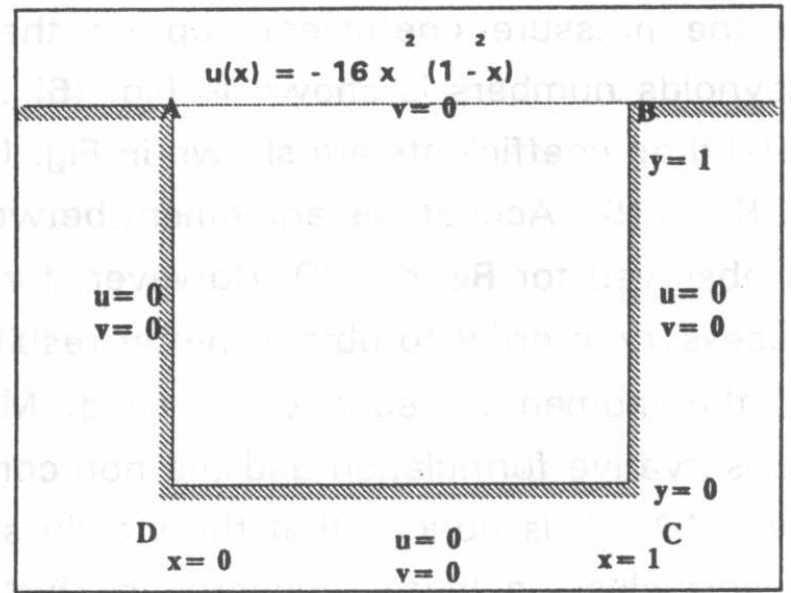

Fig. (1) Cavity boundary conditions. 


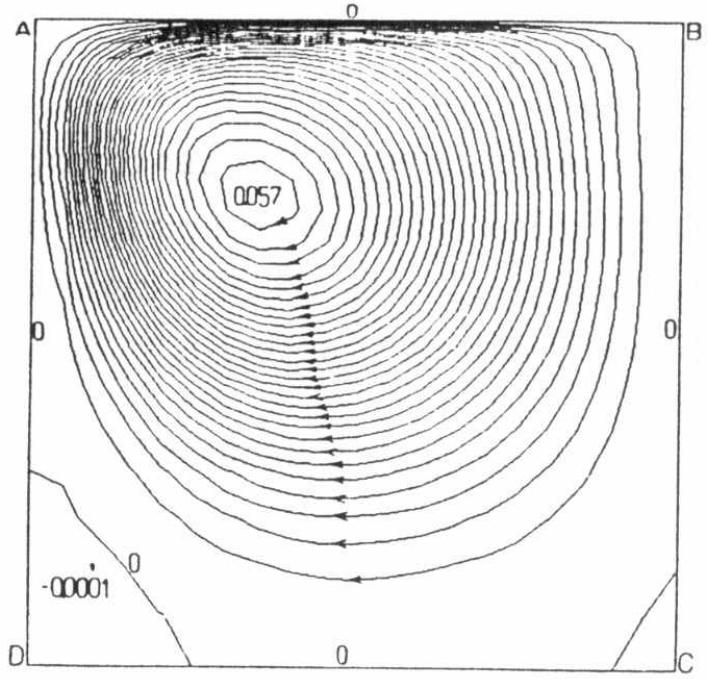

Fig. 2 Streamlines contours. (Cavity problem)

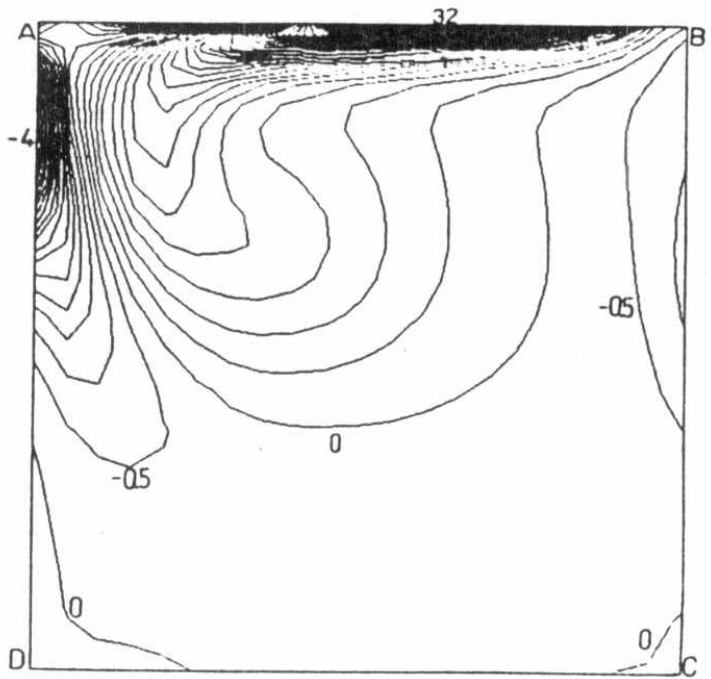

Fig. 3 Iso-vorticity lines. (Cavity problem)

In the second test case flow around a circular cylinder is considered. Computations have been performed using $41 \times 41$ grid points for five values of Reynolds number; namely $R e=60,100,200,400$, and 600 . The streamlines for two values of Reynolds number ( $R e=60$ and 600$)$ are illustrated in Figs. (4.a) and (4.b). It is noticed that, as the Reynolds number increases, the distance of the wake behind the cylinder becomes longer and the separation point moves further upstream as shown in Fig. (5) where a comparison with other Numerical and experimental results [13-15] is illustrated. The distribution of the pressure coefficient, $C p$ on the surface of the cylinder for various Reynolds numbers is shown in Fig. (6). The calculated pressure, friction and total drag coefficients are shown in Fig. (7) together with the experimental data of Ref. [15]. Acceptable agreement between calculated and experimental values is observed for $\operatorname{Re}<400$. However, for $\operatorname{Re}>400$, a finer grid seems to be necessary in order to obtain better results. In all cases the conservative form of the momentum equation is used. Meanwhile a comparison between this conservative formulation and the non-conservative one has been performed for $\mathbf{R e}=400$. It is noticed that the steady state solutions are practically identical. Nevertheless, a better convergence history is observed for the conservative formulation as shown in Fig. (8). 

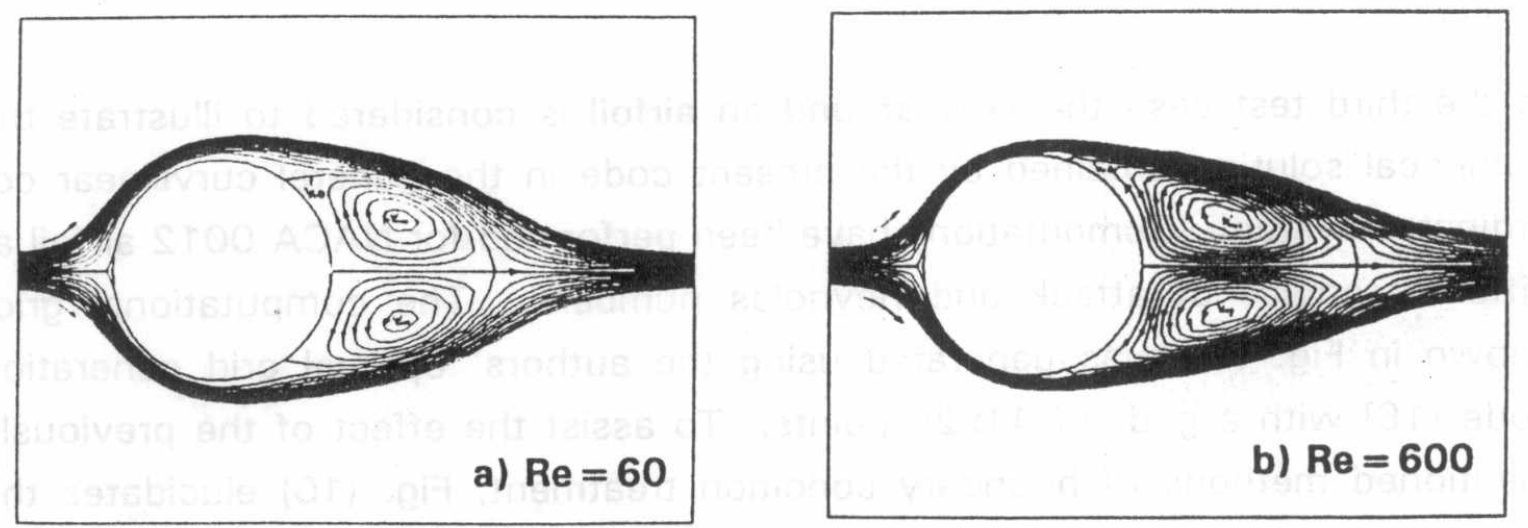

Fig. 4 Streamline pattern - flow around circular cylinder

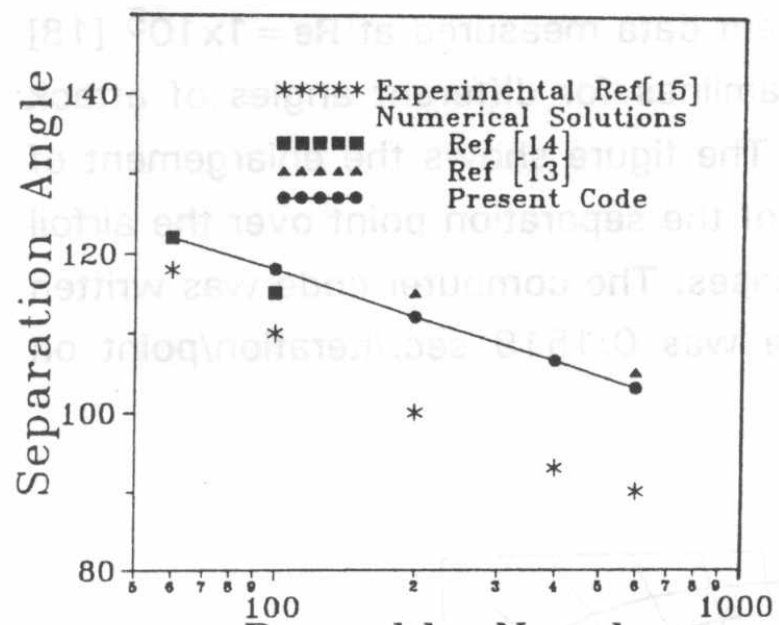

Reynolds Number

Fig. 5 Seperation angle measured from stagnation point. (circular cylinder)

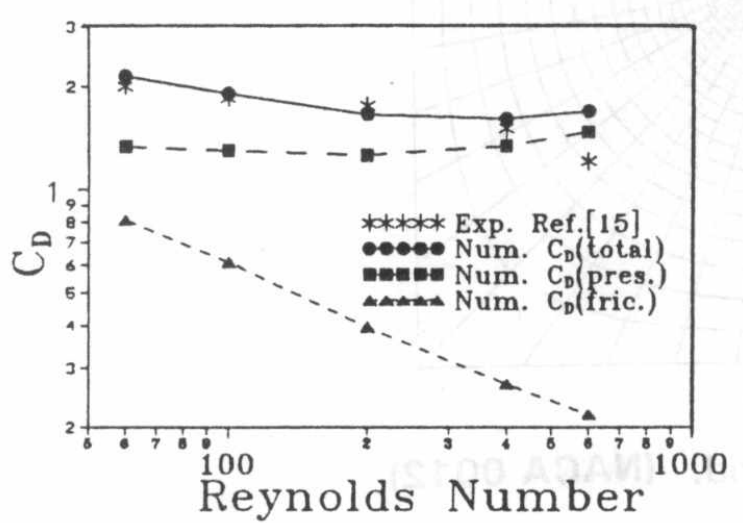

Fig. 7 Drag coefficient versus Reynolds number.

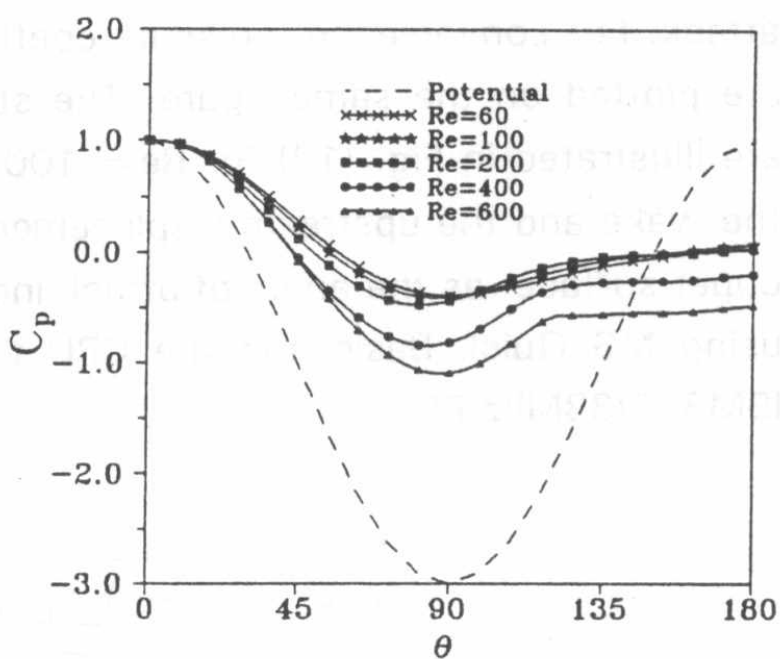

Fig. 6 Pressure coefficient distribution. (circular cylinder)

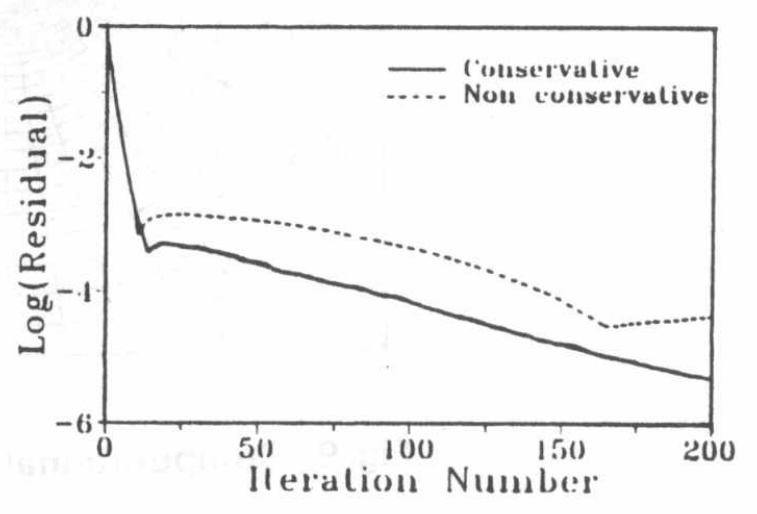

Fig. 8 Convergence history. (circular cylinder) 
In the third test case the flow around an airfoil is considered to illustrate the numerical solution obtained by the present code in the general curvilinear coordinates system. Computations have been performed for NACA 0012 airfoil at different angles of attack and Reynolds numbers. The computational grid, shown in Fig. (9), was generated using the authors' optimal grid generation code [16] with a grid of $41 \times 25$ points. To assist the effect of the previously mentioned methods of boundary condition treatment, Fig. (10) elucidates the convergence histories obtained using the first and second methods for image points calculations. Fig. (11) compares the calculated pressure coefficient with the numerical results of Ref. [17] for the case of $R e=400$ and zero angle of attack. For convenience, pressure coefficient data measured at $R \mathbf{e}=1 \times 10^{5}$ [18] are plotted on the same figure. The streamlines for different angles of attack are illustrated in Fig. (12) for $\mathbf{R e}=1000$. The figure shows the enlargement of the wake and the upstream displacement of the separation point over the airfoil upper surface as the angle of attack increases. The computer code was written using MS Quick Basic and the CPU time was $0.1519 \mathrm{sec}$./iteration/point on IBM386/33Mhz PC.

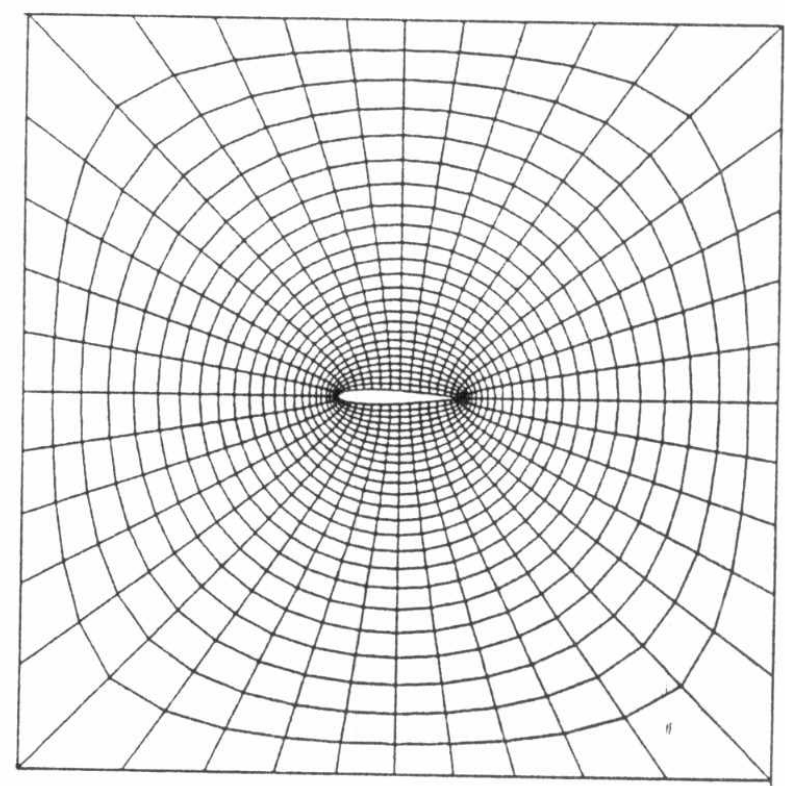

Fig. 9 Computational grid. (NACA 0012) 


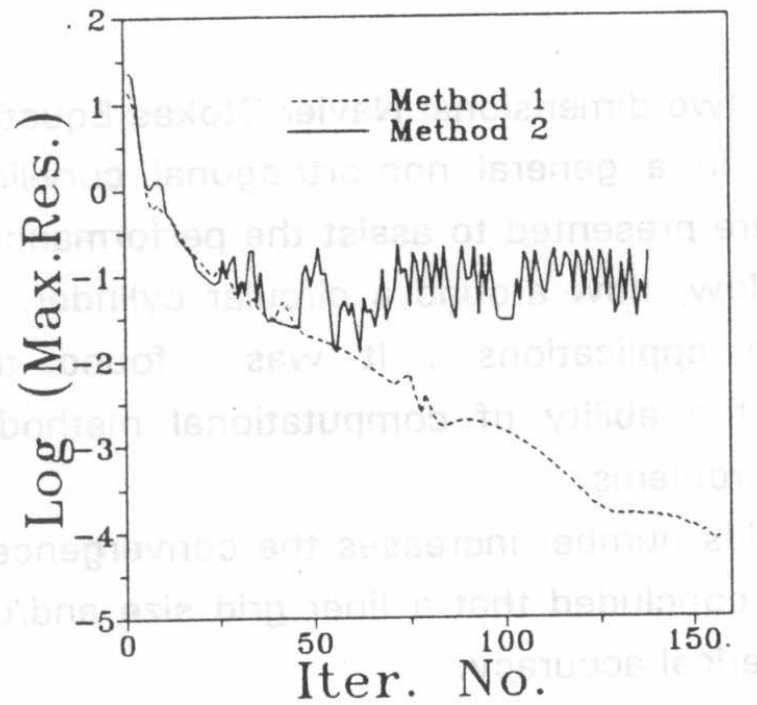

Fig. 10 Convergence history. (NACA 0012)

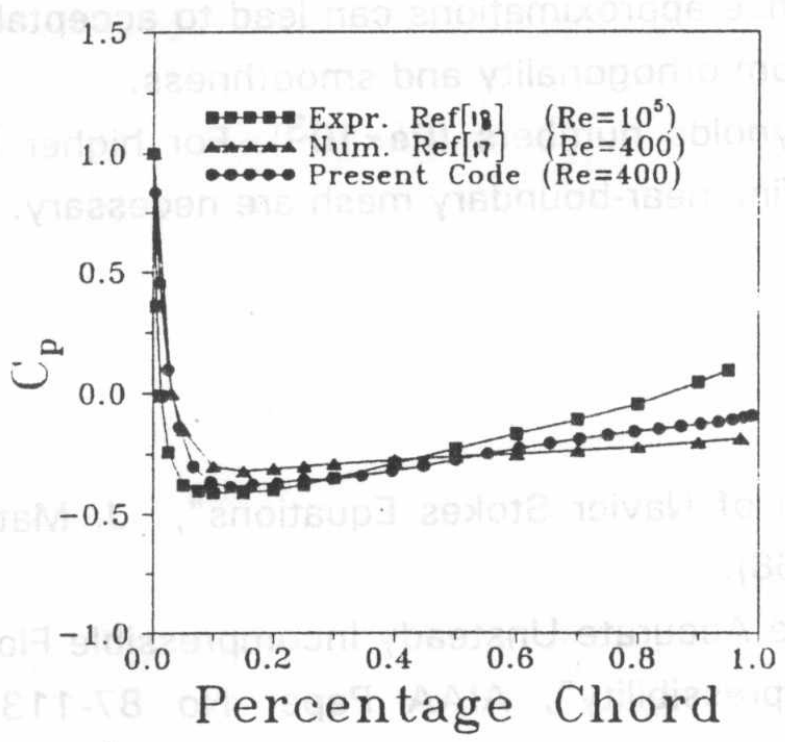

Fig. 11 Pressure distribution. (NACA-0012, zero angle of attack )

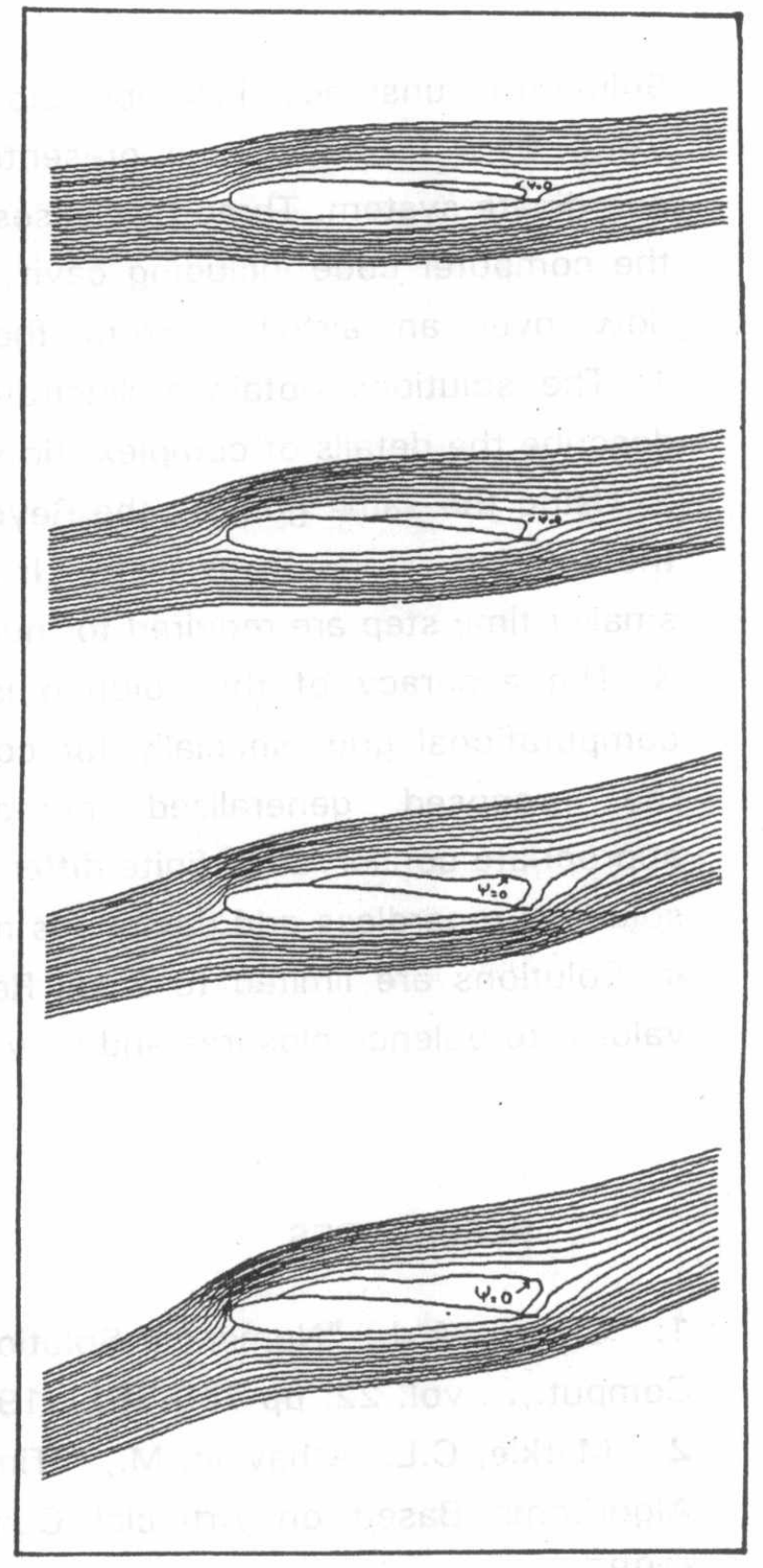

Fig. 12 Streamline pattern for angles of attack $=5^{\circ}, 10^{\circ}, \quad 15^{\circ}$ and $20^{\circ}$. 


\section{CONCLUSIONS}

Solution of unsteady, incompressible, two-dimensional Navier-Stokes Equations using $\boldsymbol{\Psi}-\boldsymbol{\omega}$ formulation is presented in a general non-orthogonal curvilinear coordinate system. Three test cases are presented to assist the performance of the computer code including cavity flow, flow around a circular cylinder, and flow over an airfoil. From these applications, It was found that: 1- The solutions obtained illustrate the ability of computational method to describe the details of complex flow problems.

2- Using the same grid, as the Reynolds number increases the convergence of the solution starts to oscillate. It is concluded that a finer grid size and/or a smaller time step are required for numerical accuracy.

3- The accuracy of the solution is highly dependent on the quality of the computational grid, specially for complex geometry problems. Nevertheless, the proposed generalized non-orthogonal curvilinear formulation with appropriate conservative finite difference approximations can lead to acceptable solutions regardless grid deviations from orhogonality and smoothness.

4- Solutions are limited to small Reynolds numbers ( $\operatorname{Re} \sim 10^{3}$ ). For higher $\mathbf{R e}$ values, turbulence closures and very fine near-boundary mesh are necessary.

\section{REFERENCES}

1. Chorin, A.J., "Numerical Solution of Navier Stokes Equations", J. Math. Comput... , vol. 22, pp 745-762, (1968).

2. Merkle, C.L., Athavale, M., " Time Accurate Unsteady Incompressible Flow Algorithms Based on Artificial Compressibility", AIAA Paper No 87-1137, (1987).

3. Rogers, S.E., Kwak, D. and Kiris, C., "Steady and Unsteady Solutions of the Incompressible Navier Stokes Equations", AIAA Journal, Vol. 29, No. 4, pp. 603-610, (1991).

4. Hanel, D. and Breuer, M., " Numerical Solution of the Incompressible Navier Stokes Equations for Unsteady Three Dimensional Flow", Int. Conf. Fluid Mechanics, ICFM3, pp. 1111-1124, (1990).

5. Harlow, F.H. and Welch, J.E., "Numerical Calculation of Time-Dependent Viscous Incompressible Flow of Fluid with Free Surface", Physics of Fluids, Vol. 8, No. 12, pp 2182-2189, (1965). 
6. Fasel, H.," Investigation of the Stability of Boundary Layers by a Finite Difference Model of the Navier-Stokes Equations", J. Fluid Mech., Vol.78, pp 355-383, (1972).

7. Hafez, M., Dacles, J., and Soliman, M.," A Velocity-Vorticity Method for Viscous Incompressible Flow Calculations", Proc. of the 11th Int. Conf. on Numerical Methods in Fluid Mechanics, Williamsburg, Virginia, (1989).

8. Napolitano,M. and Pascazio, G., " A Multigrid LGS Method for the Vorticity-Velocity Navier-Stokes Equations", Proc. of the 8th GAMM Conf. on Numerical Methods in Fluid Mechanics, Delft, Netherlands, (1989).

9. Chamberlian,J.P., and Liu, C.H.," Navier-Stokes Calculations for Unsteady Three Dimensional Vortical Flows in Unbounded Domains", AIAA- paper No. 84-0418, (1984).

10. Peyret, R., and Taylor D.T.," Computational Methods for Fluid Flow", Springer Series in Computational Physics, Springer-Verlag, New York,(1983). 11. Peaceman, D.W., and Rachford, H.H.," The Numerical Solution of Parabolic and Elliptic Differential Equations", J. Soc. Indust. Appl. Math., Vol.3, pp. 28, (1955).

12. Douglas, J., and Gunn, J.E.," A General Formulation of Alternating Direction Methods", Part I, Parabolic and Hyperbolic Problems", Numerical Mathematics, Vol.6, pp. 428-453, (1964).

13. Thoman, D.C. and Szewczyk, A.A.,"Time-Dependent Viscous Flow over a Circular Cylinder", Phys.Fluids Supp.II, 76, pp. 76-86, (1969).

14. Son, J.S. and Hanratty, T.J., "Numerical Solution for the Flow around Cylinder at Reynolds Numbers of 40, 200 and 500", J.Fluid Mech., Vol. 35, part 2, pp. 369-386, (1969).

15. Schlichting, H., "Boundary - Layer Theory" , Mc Graw Hill, Co., NewYork (1968).

16. Abdelrahman, M.M. and Al - Bahi, A.M., " Optimal Smooth and Orthogonal Grid Generation", AIAA - ISABE Paper No. 91-7033, (1991).

17. Giannakoglou, K., Lymberopoulos, N., Chaviaropoulos, P. and Papailiou, K. D., " A Navier Stokes Vorticity-Stream function Formulation For TwoDimensional Flows", Fourth International Conference of Fluid Mechanics, ICFM4, Alexandria, pp.133-144, April (1992).

18. Straus, J., Renzoni, P. and Mayle, R.E., "Airfoil Pressure Measurements During a Blade Vortex Interaction and a Comparison with Theory", AIAA Journal, Vol. 28, No. 2, pp. 222-228, (1990). 